\title{
Ag nanowires functionalized cellulose textiles for supercapacitor and photothermal conversion
}

\author{
Yang Yanga ${ }^{\mathrm{a}}$, Runcang Sun ${ }^{\mathrm{a}, \mathrm{b}}$, Xiaohui Wang ${ }^{\mathrm{a}, \mathrm{c}, *}$
}

\begin{abstract}
:
Smart textiles are attracting increasing attention due to their tremendous applications in wearable devices. Herein, we report a flexible and conductive textile prepared via simple "dipping and drying" method with Ag nanowires (Ag NWs) ink. The obtained Ag NWs/cellulose textiles have very high conductivity(sheet resistance $0.2 \Omega / \mathrm{sq}$ ) and excellent chemical and mechanical stabilities, showing great potential in wearable energy storage and photothermal conversion. The supercapacitor made from the Ag NWs/cellulose textile showed a higher capacitance (159 $\left.\mathrm{F} \mathrm{g}^{-1}\right)$ than the textiles functionalized with single-walled carbon nanotube (SCNT) and graphene. Besides, they presented excellent photothermal conversion property with about $50 \%$ improvement in temperature compared with pure textile. These multifunctional textiles would provide new opportunities for flexible and wearable electronics and sensors.
\end{abstract}

Keywords: electronic textiles, Ag nanowires, supercapacitor, photothermal conversion 


\section{Introduction}

Emerging research on smart textiles has revolutionized the use of textile as a wearable platform for tremendous applications, including military garment devices, personal electronics and so on.[1, 2]Recently, some pioneer researchers reported the fabrication of flexible, lightweight and wearable power conversion and storage textile devices by relatively high-throughput, commercially viable processes, which greatly feathered the industrialization of smart textile. For example, Cui et al. fabricated conductive textiles with a "dipping and drying" process using SCNT ink. [3, 4] Xue et al. reported a flexible cotton electrode via a "brush-coating and drying" process with graphene oxide.[5]Although great potential has been revealed, the electrical conductivity of those functional textiles still need improvement.

Silver nanowires are highly conductive nanomaterials with good antimicrobial activities, which make them promise candidates for preparing smart textile. Recently, some researches have been coating silver nanowires on cotton or papers, and proved that silver nanowires could cooperate with cellulose fiber due to the hydrogen bonding between them.[6-8]However, it is still a great challenge to achieve high conductivity while keeping good mechanical and chemical durabilities of the composite textiles, and the latters are critical for device fabrication and high-speed roll to roll fabrication.[9]. Therefore, there's an urgent need to develop efficient and feasible method for stably functionalizing textile substrates with Ag NWs.

Here, we report a novel multifunctional smart textile with uniformly coated Ag NWs via an extremely simple "dipping and drying" process. And their mechanical and chemical durabilities have been detected in detail. The three-dimensional high-surface-area characteristic of such textile facilitates the absorption of $\mathrm{Ag} \mathrm{NWs,} \mathrm{which} \mathrm{enables} \mathrm{high} \mathrm{electrical} \mathrm{and} \mathrm{electrochemical}$ performance of textile supercapacitors and improved photothermal conversion ability. This Ag NWs functionalized smart textile may find application in wearable energy storage and photothermal conversion device.

\section{Experimental method}

Ag NWs were synthesized according to the polyol process.[10]The highly conductive Ag NWs coated textiles was achieved through a simple dipping and drying process. Specifically, a piece of cotton sheet $(2 \mathrm{~cm} \times 4 \mathrm{~cm} \times 200 \mu \mathrm{m}$, purchased from the nearest supermarket $)$ was dipped into the $\mathrm{Ag} \mathrm{NWs} /$ ethanol ink for $3 \mathrm{~min}$. Then the wet textile was dried in a pre-heated oven at $105^{\circ} \mathrm{C}$ for 1 h. This procedure was repeated for several times to evaluate the effect of recycle time on the textile properties.

The morphology of Ag NWs and textile fibers was observed with a scanning electron microscope (SEM, Santa Clare, CA, USA).The sheet resistance (Rs) of the Ag NWs coated textiles was measured by a four-probe method (RTS-8, 4 PROBE TECH, China). The electrochemical tests were performed with a standard three electrode set-up, in which $\mathrm{Ag} / \mathrm{AgCl}$ was used as reference electrode, Pt foil as counter electrode and $\mathrm{Ag} \mathrm{NWs/textiles} \mathrm{as} \mathrm{working} \mathrm{electrode,} \mathrm{foamed} \mathrm{nickel}$ attached to the textile by silver paste as current collectors in $6 \mathrm{M} \mathrm{KOH}$. The electrochemical properties were investigated using an electrochemical working station (CHI660D, Shanghai, China). Photothermal conversion properties were tested by an $808 \mathrm{~nm}$ infrared diode laser with a power density of $4 \mathrm{~W} \mathrm{~cm}^{-2}$ (Changchun New Industries Optoelectronics Technology Co., Ltd.) and a thermometer (UNI-T 1310, UNI-T Electronic Crop.). 


\section{Results and discussion}
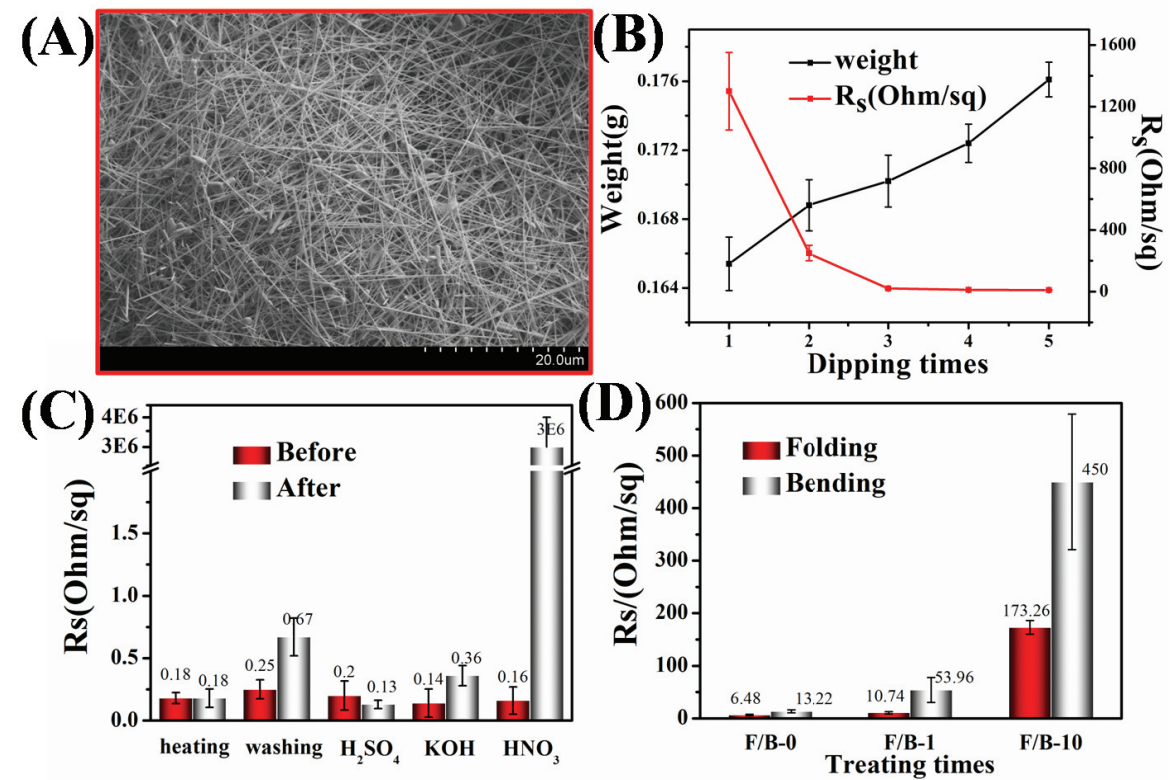

Figure 1.(A):SEM image of purified Ag NWs. (B):Rs and weight of fabric sheet after Ag NWs coating. (C)(D):Mechanical and chemical stability of fabric sheets.

The synthesized Ag NWs, that can be uniformly dispersed in ethanol to form colloidal-stable ink, have high length-diameter ratio with small mean diameter $(<50 \mathrm{~nm})$ and large average length $(>20$ $\mu \mathrm{m})$ (Figure 1A).Those Ag NWs functionalized textiles exhibited excellent electrical stability. As shown in Figure 1B, the $\mathrm{R}_{S}$ of the fabric initially reduces sharply with the adding dipping times in Ag NWs ink, but tends to keep constant after three times dipping. A significantly low $R_{s}$ of $<0.2$ $\Omega / \mathrm{sq}$ was achieved with only three dipping cycles, which is much less than those textiles functionalized with carbon materials or silver nanowires.[5, 11]On the other hand, the overall weight of the textile kept increasing with the adding dipping cycles indicating the textile has the ability to hold much more Ag NWs than necessary. This property permits them to accommodate other functional materials or molecules, such as carbon materials and monomer of polymers..

The Ag NWs functionalized textiles demonstrate excellent mechanical and chemo-physical resistance performance as illustrated in Figure 1C and D. When the conductive textile was exposed to thermal treatment at $105^{\circ} \mathrm{C}$ for $3 \mathrm{~h}, 2 \mathrm{M} \mathrm{H}_{2} \mathrm{SO}_{4}$ for $15 \mathrm{~min}$ and $2 \mathrm{M} \mathrm{KOH}$ for $15 \mathrm{~min}$, $\mathrm{R}_{\mathrm{S}}$ showed a negligible change. Only concentrated nitric acid resulted in a dramatically increase of $R_{s}$, which is probably due to the reaction between the Ag NWs and nitric acid. Thus, if those Ag NWs/textiles were used as energy storage devices, their excellent chemical stability would allow a wide range of operations. The conductive textile also presented good stability upon repeated washing treatments. After washing and squeezing gently for 5 times, $R_{s}$ was still as low as $0.67 \Omega /$ sq. Besides, the operation of bending and folding could not significantly change the conductivity of $\mathrm{Ag} \mathrm{NWs/textiles,} \mathrm{neither.} \mathrm{But} \mathrm{when} \mathrm{the} \mathrm{textiles} \mathrm{were} \mathrm{bended/folded} \mathrm{for}>10$ times, $\mathrm{R}_{\mathrm{s}}$ increased sharply possibly due to the exfoliation or break of Ag NWs. The superior mechanical adhesion of Ag NWs is essential for high-speed roll to roll fabrication and energy storage device stability.

The SEM images as shown in Figure 2A and B revealed uniform coating of Ag NWs on cellulose fibers, in which every fiber was tightly wrapped by Ag NWs. The macroporous structure of the 
textiles and the hydrogen bonds between cellulose fibers and polyvinyl pyrrolidone(PVP) coated nanowires probably result in the strong adhesion. Such a uniform coating of Ag NWs could account for the high electrical conductivity and mechanical flexibility of the textiles, which were also evidenced by a bright LED bulb lightened by an Ag NWs/textile embedded circuit(Figure 2C).
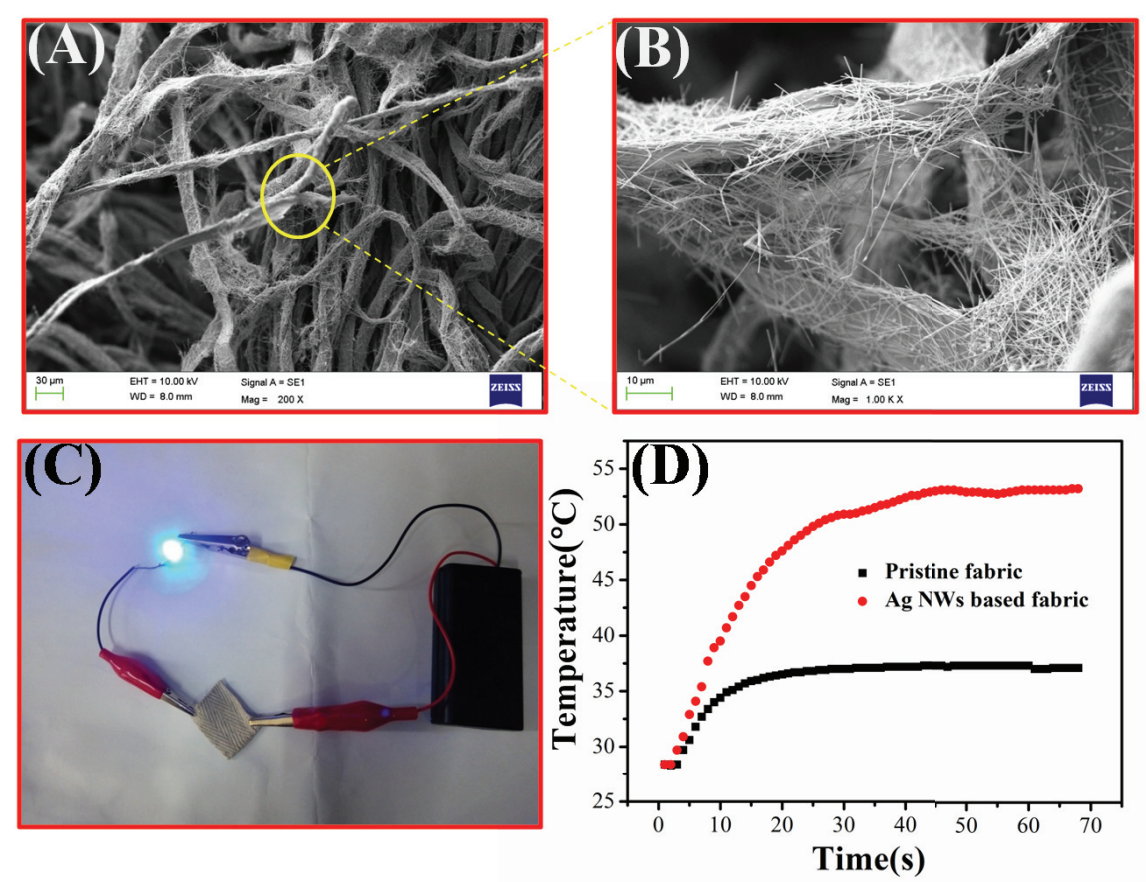

Figure 2. (A) and (B): SEM images of Ag NWs fabrics. (C): The conductive performance of the Ag NWs based fabric.(D):Temperature profiles of pristine and $\mathrm{Ag}$ NWs coated fabric under laser irradiation.

We also found the Ag NWs/textile have unique photothermal conversion property. As shown in Figure 2D, the Ag NWs/textile exhibited a sharp increase in temperature with prolonged exposure time under $808 \mathrm{~nm}$ laser, and approached a plateau after about 1 min irradiation. This result indicated that the Ag NWs/textile could rapidly convert laser energy to environmental heat due to the electron-phonon and phonon-phonon process[12]. This property may find application in photothermal therapy. 

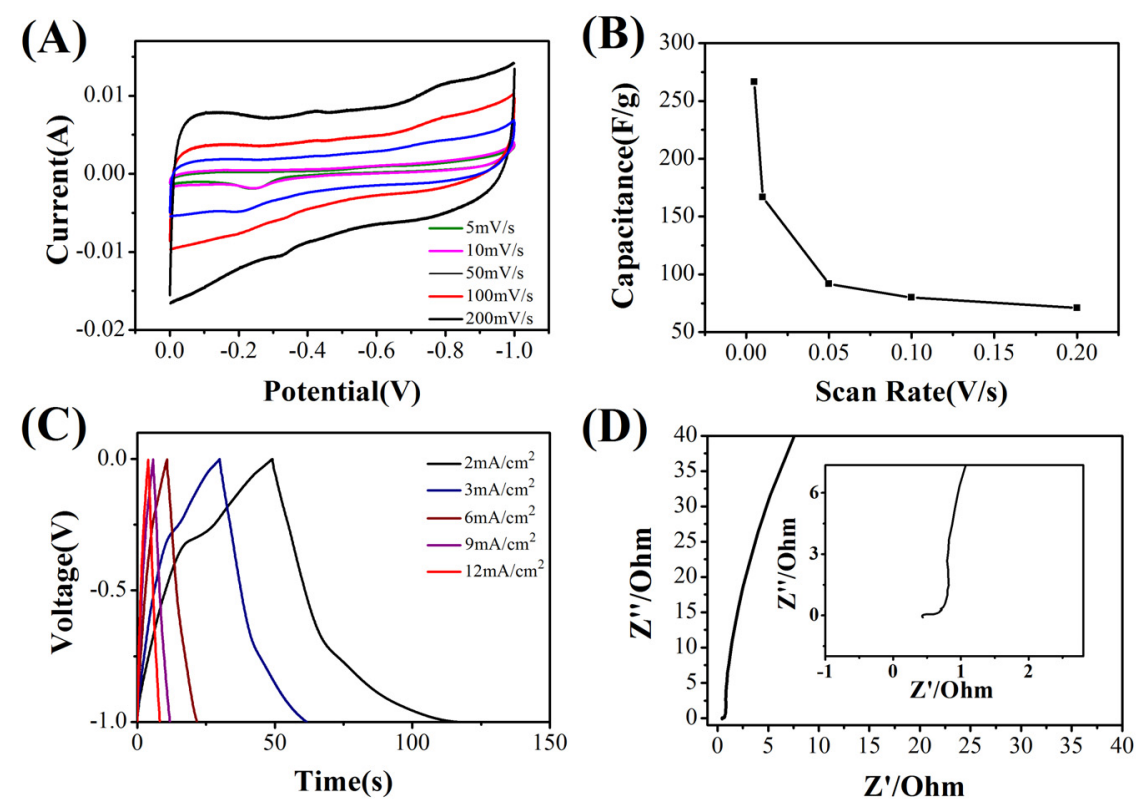

Figure 3. A: Cyclic voltammetry $(\mathrm{CV})$ curves of electrode at different scan rates. B: Specific capacitance at different scan rate. C: Galvanostatic $(\mathrm{GV})$ constant-current charge/discharge curves at various current densities. D: Impedance spectroscopy of the electrode.

The porous structure of the conductive textile would facilitate the easy access of electrolyte ions to the Ag NWs, which prompted us to investigate their application in supercapacitors. The electrochemical performance was shown in Figure 3. The CV curves showed excellent capacitive behavior with quasi-rectangular shape, indicating the stable and high electrochemical performance of the electrode. The specific capacitance decreased gradually with increasing scan rate and achieved $159 \mathrm{~F} \mathrm{~g}^{-1}$ at $10 \mathrm{mV} \mathrm{s}^{-1}$, which was much better than the graphene-cotton cloth and SCNT/ textiles.[3,5] This may be attributed to the significantly improved conductivity of the Ag NWs/ textiles. The GV curves had a very symmetric nature at current densities ranging from 9 to $12 \mathrm{~mA}$ $\mathrm{cm}^{-2}$, which indicated a rapid current-voltage response and good electrochemical capacitive behavior. However, when the current densities decreased, a small sloping potential profile with a potential plateau that arose from the Faradaic reaction was observed in these curves. [13]Impedance spectroscopy was used to further study the electrochemical behaviors. In the low frequency region of Nyquist plots, the plot is nearly perpendicular to the real axis, which indicated the purely capacitive behavior.[14]In the high frequency region (Figure 3D inset), both of the equivalent series resistance and the charge-transfer resistance[15] showed a very low value, which indicated the highly conductive Ag NWs/textiles favored accessing of ions due to the good wettability and porous property of the textile fibers.

\section{Conclusions}

In summary, we report a simple and efficient method to fabricate highly conductive multifunctional $\mathrm{Ag} \mathrm{NWs/textiles.} \mathrm{The} \mathrm{as-prepared} \mathrm{Ag} \mathrm{NWs} /$ textiles have very low $\mathrm{R}_{\mathrm{s}}(0.2 \Omega / \mathrm{sq})$, good electronic stability, good electrochemical performance and excellent photothermal conversion properties, showing great potential in wearable energy storage, optical heating and photothermal therapy.

\section{Acknowledgement}


This work was supported by the National Science Foundation of China (51673072), the Independent Study Projects of the State Key Laboratory of Pulp and Paper Engineering (2016TS01), the Science and Technology Program of Guangzhou, China (201504010033) and the New Century Excellent Talents in University (NCET-13-0215).

\section{References}

[1] L. Bao, X. Li, Towards Textile Energy Storage from Cotton T-Shirts, Adv. Mater. 24(24) (2012) 3246-3252.

[2] L. Liu, Y. Yu, C. Yan, K. Li, Z. Zheng, Wearable energy-dense and power-dense supercapacitor yarns enabled by scalable graphene-metallic textile composite electrodes, Nat Commun 6 (2015).

[3] L. Hu, M. Pasta, F.L. Mantia, L. Cui, S. Jeong, H.D. Deshazer, J.W. Choi, S.M. Han, Y. Cui, Stretchable, porous, and conductive energy textiles, Nano Lett. 10(2) (2010) 708-14.

[4] L. Hu, J.W. Choi, Y. Yang, Y. Cui, Conductive fibrous materials, Google Patents, 2015.

[5] W.-w. Liu, X.-b. Yan, J.-w. Lang, C. Peng, Q.-j. Xue, Flexible and conductive nanocomposite electrode based on graphene sheets and cotton cloth for supercapacitor, J. Mater. Chem. 22(33) (2012) 17245 .

[6] Y. Wei, S. Chen, Y. Lin, X. Yuan, L. Liu, Silver nanowires coated on cotton for flexible pressure sensors, J Mater Chem C 4(5) (2016) 935-943.

[7] H. Kopeinik, R. Schennach, J. Gallik, H. Plank, B. Friedel, Photodiodes based on wood pulp fiber networks, Cellulose 22(5) (2015) 3425-3434.

[8] T.-W. Lee, S.-E. Lee, Y.G. Jeong, Highly Effective Electromagnetic Interference Shielding Materials based on Silver Nanowire/Cellulose Papers, ACS Applied Materials \& Interfaces 8(20) (2016) 13123-13132.

[9] Y. Yao, J. Tao, J. Zou, B. Zhang, T. Li, J. Dai, M. Zhu, S. Wang, K.K. Fu, D. Henderson, Light management in plastic-paper hybrid substrate towards high-performance optoelectronics, Energy \& Environmental Science (2016).

[10] M. Li, M. Jing, Z. Wang, B. Li, X. Shen, Controllable Growth of Superfine Silver Nanowires by Self-Seeding Polyol Process, Journal of Nanoscience and Nanotechnology 15(8) (2015) 6088-6093.

[11] M.R. Nateghi, M. Shateri-Khalilabad, Silver nanowire-functionalized cotton fabric, Carbohydr. Polym. 117 (2015) 160-168.

[12] J. Zhou, L. Meng, Q. Lu, Core@shell nanostructures for photothermal conversion: Tunable noble metal nanoshells on cross-linked polymer submicrospheres, J. Mater. Chem. 20(26) (2010) 5493.

[13] X. Lu, T. Zhai, X. Zhang, Y. Shen, L. Yuan, B. Hu, L. Gong, J. Chen, Y. Gao, J. Zhou, Y. Tong, Z.L. Wang, WO3-x@Au@MnO2 Core-Shell Nanowires on Carbon Fabric for High-Performance Flexible Supercapacitors, Adv. Mater. 24(7) (2012) 938-944.

[14] H. Wang, B. Zhu, W. Jiang, Y. Yang, W.R. Leow, H. Wang, X. Chen, A Mechanically and Electrically Self-Healing Supercapacitor, Adv. Mater. 26(22) (2014) 3638-3643.

[15] Z. Weng, Y. Su, D.W. Wang, F. Li, J.H. Du, H.M. Cheng, Graphene-Cellulose Paper Flexible Supercapacitors, Advanced Energy Materials 1(5) (2011) 917-922. 